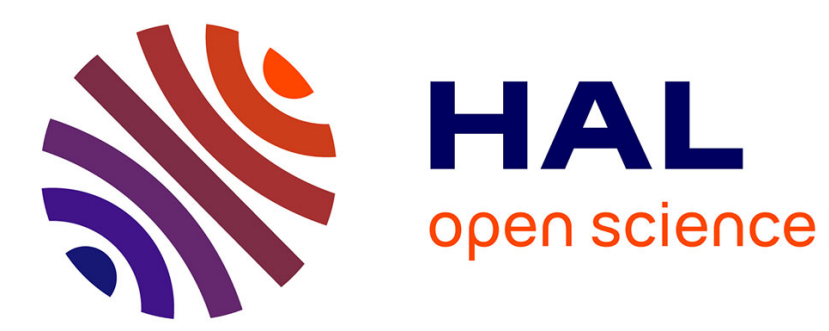

\title{
AGRICULTURE DE SERVICE : QUELQUES ÉLÉMENTS D'ANALYSE ÉCONOMIQUE
}

\author{
Olivier Aznar
}

\section{To cite this version:}

Olivier Aznar. AGRICULTURE DE SERVICE: QUELQUES ÉLÉMENTS D'ANALYSE

ÉCONOMIQUE. Pour, revue du Groupe Ruralités, Éducation et Politiques, 2014, 221. hal-02096095

\section{HAL Id: hal-02096095 \\ https://hal.uca.fr/hal-02096095}

Submitted on 11 Apr 2019

HAL is a multi-disciplinary open access archive for the deposit and dissemination of scientific research documents, whether they are published or not. The documents may come from teaching and research institutions in France or abroad, or from public or private research centers.
L'archive ouverte pluridisciplinaire HAL, est destinée au dépôt et à la diffusion de documents scientifiques de niveau recherche, publiés ou non, émanant des établissements d'enseignement et de recherche français ou étrangers, des laboratoires publics ou privés. 


\section{AGRICULTURE DE SERVICE : QUELQUES ÉLÉMENTS D'ANALYSE ÉCONOMIQUE}

Olivier Aznar

GREP | « Pour »

2014/1 N²21 | pages 79 à 86

ISSN 0245-9442

Article disponible en ligne à l'adresse :

https://www.cairn.info/revue-pour-2014-1-page-79.htm

Distribution électronique Cairn.info pour GREP.

(C) GREP. Tous droits réservés pour tous pays.

La reproduction ou représentation de cet article, notamment par photocopie, n'est autorisée que dans les limites des conditions générales d'utilisation du site ou, le cas échéant, des conditions générales de la licence souscrite par votre établissement. Toute autre reproduction ou représentation, en tout ou partie, sous quelque forme et de quelque manière que ce soit, est interdite sauf accord préalable et écrit de l'éditeur, en dehors des cas prévus par la législation en vigueur en France. Il est précisé que son stockage dans une base de données est également interdit. 


\section{Olivier AZNAR}

VetAgro Sup

E-mail : olivier.aznaravetagro-sup.fr

\section{Agriculture de service : quelques éléments d'analyse économique}

\section{Introduction}

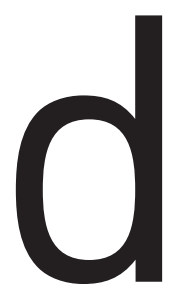

e plus en plus, on parle des « services » fournis, produits ou rendus par l'agriculture. Il s'agit par exemple de vente directe, d'agro-tourisme ou encore de services environnementaux. À notre sens, cette formulation générique de «services» revêt en fait des réalités différentes. Ainsi, l'objet de cet article ${ }^{1}$ consiste à lire les services fournis par l'agriculture avec un cadre d'analyse issu de l'économie des services. Nous pourrons ainsi mieux comprendre les différentes réalités que recouvre la notion d'agriculture de service. Nous tenterons d'en tirer des éléments sur le rôle des politiques publiques vis-à-vis de l'agriculture de service. 


\section{Caractéristiques économiques d'un service}

\section{Quelles définitions des services?}

Il existe plusieurs manières de définir les services, selon la focale retenue. À la suite des travaux pionniers de Clark (1940), les services ont été assimilés aux activités tertiaires et définis par défaut comme englobant toutes les activités ne pouvant être classées ni dans le secteur primaire ni dans le secteur secondaire. En référence à ce cadre, des délimitations des services sont couramment produites. Par exemple celle utilisée par l'INSEE comprend les transports, l'administration, le commerce, les activités financières et immobilières, les services aux entreprises et aux particuliers, l'éducation, la santé et l'action sociale. D'autres définitions ont été formulées pour circonscrire le domaine des services en cherchant des critères discriminants tels que la faible productivité ou le caractère immatériel de la prestation mais, dans le cas des services de l'agriculture, chacune de ces définitions souffre de trop d'exceptions pour être réellement satisfaisante. Une première approche (cf. tableau 1), celle de l'économie des services (Gadrey, 2003) met en scène ce que l'on peut appeler un « service-activité ». Dans ce cadre, un service est toujours une relation orientée, conçue de façon intentionnelle, en vue de permettre un usage d'un support par un (ou plusieurs) usager(s). Le service instruit ici une relation contractuelle entre deux agents. Ensuite, le service implique une relation d'échange et de contrepartie (monétaire ou non) entre les deux agents. Il faut préciser que le produit final de cette opération ne peut pas être vendu indépendamment du support. Ce support peut être un bien, un individu ou encore une organisation, mais il reste toujours indissociable de la prestation de service. Par exemple, la gestion de l'eau ou des déchets tend à être de plus en plus appréhendée en termes d'activités produisant des services environnementaux. Dans le cas du traitement de l'eau (assainissement), l'eau est un bien collectif sur lequel un opérateur (une collectivité locale ou une entreprise) va conduire un certain nombre d'opérations afin de la rendre propre. Le service rendu (service d'épuration) est considéré dans ce cadre comme un service environnemental dans le sens d'un service qui concerne le secteur économique de l'environnement - et s'inscrit dans notre catégorie de « service-activité ».

Une deuxième approche, s'inscrivant dans les travaux de Lancaster (1966) et de l'économie des fonctionnalités ${ }^{2}$, permet de qualifier les services de « service-produit joint » (cf. tableau 1). La logique retenue est celle de l'intégration de nouvelles fonctionnalités dans le produit lagricole, par exemple). Suivant cette perspective, le produit agricole ne se réduit plus à un bien tangible, il doit garantir aussi le respect des processus de production et des types d'approvisionnement en amont Iressources génétiques, alimentation animale, produits vétérinaires, semences, 
produits phytosanitaires, etc.). Au cours des dernières années la croissance du contenu en informations que le produit agricole doit apporter, témoigne d'un tel changement structurel : le produit agricole se «tertiarise ». Dans la mesure où ces différentes dimensions de l'offre agricole sont conçues et intégrées au produit pour répondre à la demande du consommateur, le produit agricole devient une « solution agricole » intégrant biens et services. Ce qui est produit et vendu, c'est un bouquet de services et de biens complémentaires (Moati, 2008 ; Jeanneaux et al., 2011), par exemple du chocolat aux noix de cajou, produit en agriculture biologique et issu du commerce équitable. La rémunération des services incorporés au produit est assurée par le consentement du consommateur à payer davantage pour ce produit et ses services joints. Le consommateur rémunère ainsi directement un engagement de moyens du producteur.

\section{Tableau 1 : Typologie des services}

\begin{tabular}{llll}
\hline \multicolumn{1}{c}{ Type } & \multicolumn{1}{c}{ Définition } & $\begin{array}{c}\text { Type d'approche } \\
\text { mobilisée }\end{array}$ & Lien avec des dispositifs \\
\hline «Service & $\begin{array}{l}\text { Opération réalisée } \\
\text { par un prestataire à la } \\
\text { demande d'un usager }\end{array}$ & $\begin{array}{l}\text { Économie des } \\
\text { services }\end{array}$ & $\begin{array}{l}\text { Incitation, organisation du } \\
\text { marché loffre et demande) }\end{array}$ \\
\hline ou contractualisation
\end{tabular}

\section{Définition de l'agriculture de service}

Dans notre acception, la production de services par l'agriculture implique la modification ou le maintien en l'état d'un support et/ou la mise à disposition de capacités techniques et humaines par l'agriculteur. La relation de service est orientée vers la réponse à une demande, portée par des usagers finaux ou par des représentants de ces usagers.

Ce service est souvent articulé avec une activité principale relevant de la production de bien agricole. Il s'agit là d'une caractéristique importante de l'agriculture de service : elle repose généralement sur des économies de gamme ${ }^{2}$ entre la production du bien agricole et la production du service.

2 Les économies de gamme sont des bénéfices qui peuvent résulter de l'association des productions, par exemple entre l'élevage et les céréales : le fumier sert d'apport organique pour les cultures de céréales. 


\section{Classement des services de l'agriculture selon la nature de leur demande}

Dans cet article, nous proposons un classement des services-prestations selon la nature de leur demande. Par «nature de la demande », nous entendons le nombre de bénéficiaires:

- s'agit-il d'un acteur individuel ?

- d'un nombre limité de bénéficiaires (par exemple un club) ?

- ou d'un service qui bénéficie (potentiellement) à tout le monde?

En fonction de ce critère de la demande de service, nous pouvons distinguer trois types de services : individuel, club ou collectif (cf. tableau 2).

Tableau 2 - Classement des services

\begin{tabular}{|c|c|c|}
\hline Type de services & Caractéristiques de la demande & Exemples \\
\hline \multirow{2}{*}{$\begin{array}{l}\text { Type } 1 \\
\text { Service } \\
\text { individuel }\end{array}$} & Demande privée des entreprises & Travail à façon chez un agriculteur \\
\hline & Demande privée des ménages & Vente directe, agro-tourisme \\
\hline $\begin{array}{l}\text { Type } 2 \\
\text { Service club }\end{array}$ & $\begin{array}{l}\text { Non rivalité de l'usage du service, } \\
\text { mais limitation de l'usage à un } \\
\text { «club » de bénéficiaires - } \\
\text { Demande souvent portée par des } \\
\text { associations ou des réseaux locaux }\end{array}$ & $\begin{array}{l}\text { Préservation de gibier pour une } \\
\text { association de chasse }\end{array}$ \\
\hline $\begin{array}{l}\text { Type } 3 \\
\text { Service collectif }\end{array}$ & $\begin{array}{l}\text { Non rivalité de l'usage du service } \\
\text { et impossibilité d'exclure les usa- } \\
\text { gers potentiels - Demande souvent } \\
\text { portée par les pouvoirs publics }\end{array}$ & $\begin{array}{l}\text { Puits de carbone, préservation de } \\
\text { la biodiversité, entretien de chemin }\end{array}$ \\
\hline
\end{tabular}

Type 1 : service individuel. On peut exclure les usagers n'ayant pas payé pour en profiter. Dans le cas d'un service privé, l'usager effectue directement la demande de service. L'intervention publique se limite à la construction éventuelle du marché et à l'organisation de ce dernier, par exemple l'édiction de normes et de règles ou le respect du droit du travail. On peut distinguer la demande privée des ménages et la demande privée des entreprises. Dans les deux cas, on parle de service individuel, car il s'agit de la réponse à une demande portée par un acteur en particulier. Type 2 : service club. Il s'agit d'un service dont l'usage par un acteur ne réduit pas la possibilité d'usage par d'autres acteurs. On parle de non-rivalité. Cependant on limite l'usage à un « club » de bénéficiaires. C'est pourquoi on parle de service club. Par exemple, une association de chasseurs commandite à un agriculteur un service environnemental en lien avec la faune. Le bénéfice du service est réservé aux membres de l'association de chasseurs. 
Type 3 : service collectif. Il est impossible d'exclure les usagers potentiels et il n'y a pas rivalité dans la consommation du service. Dans le cas d'un service collectif, un commanditaire se place souvent entre le prestataire et l'usager final. Ce commanditaire s'érige en traducteur de la demande des usagers finaux. Ce commanditaire a généralement un statut d'agent public - mais ce n'est pas une règle absolue - pour un service collectif. Par exemple, le commanditaire peut être l'État qui propose à des agriculteurs une mesure agro-environnementale visant la préservation de la biodiversité.

Cependant, les frontières entre services privés, mixtes ou collectifs ne sont pas fixes. La notion de service collectif dépend des choix politiques et des conventions sociales propres à une société et à une époque données (Kaul, 2001)

\section{Résultats empiriques sur l'offre de services par l'agriculture}

\section{Apports et limites des approches statistiques sur l'agriculture de service}

Les statistiques agricoles officielles ont été conçues pour permettre le suivi économique et politique de l'activité agricole. Comme nous l'avons présenté, le référentiel implicite est celui d'une exploitation centrée sur la production de biens. C'est pourquoi il est difficile de tirer des statistiques agricoles des conclusions sur les services fournis par l'agriculture. Ces statistiques n'ont pas été conçues dans ce but. Ainsi, peu de travaux ont quantifié le développement de l'agriculture de service. Capt et Dussol (2004) proposent une analyse des résultats du recensement de l'agriculture de 2000 quant à l'agriculture de service. L'agriculture de service se distingue également par deux caractéristiques (Capt et Dussol, 2004). D'une part, le niveau de formation des exploitants agricoles pratiquant une activité de service est supérieur à celui de la moyenne des exploitants ; d'autre part, le nombre d'emplois des exploitations pratiquant une activité de service est plus important que celui des exploitations exclusivement agricoles. Les exploitations agricoles pratiquant un service représentent un tiers de l'emploi agricole. De plus, $14 \%$ des exploitations pratiquant une activité de service ont recours à au moins un salarié. Ce ratio est plus faible pour les autres exploitations agricoles. Cela peut être relié aux caractéristiques de la production d'un service, souvent plus intensif en travail que la production d'un bien.

Il ne faut pas tirer de conclusion hâtive des travaux statistiques parce que la définition retenue pour les services n'est pas la même que dans cet article.

Notamment, elle ne tient pas compte des services mixtes et collectifs. De plus, les personnes qui abandonnent le statut d'agriculteur pour produire des services disparaissent des statistiques agricoles. Globalement, il semble que les données statistiques 
sous-estiment l'importance des services, en particulier dans le domaine de l'environnement.

\section{Relecture des exemples du dossier de la revue POUR}

Les différents exemples présentés dans le dossier de la revue POUR se situent sur un continuum entre les services-activités et les services-produits joints. Un article présente par exemple le cas de l'accueil de personnes en difficulté dans l'exploitation agricole, ce qui est typiquement un cas de service activité. Il s'agit d'un service à dimension collective. D'autres exemples du dossier concernent la transformation de produits dans les exploitations. Il s'agit typiquement de services - produits joints, car les exploitants cherchent à améliorer le contenu en service du produit qu'ils proposent. Il s'agit ici de services individuels. La réalisation de services par les agriculteurs peut s'initier par une opération conduite au sein de l'exploitation, puis se développer progressivement, en dépassant le cadre de l'exploitation. Il s'agit par exemple de l'initiative «Bro an Are » où, au-delà du magasin collectif, les agriculteurs ont initié une démarche de développement local (création d'un Centre d'aide par le travail et d'un écomusée notamment).

\section{Un cadre institutionnel peu adapté mais de nombreuses politiques en faveur de l'agriculture de service}

Au-delà d'une meilleure approche statistique, d'une meilleure connaissance de l'activité et de ses évolutions, en quoi les politiques publiques sont-elles concernées ? Les lois d'orientation agricole ont élargi la définition juridique des exploitations agricoles, intégrant progressivement les services que ces dernières peuvent rendre. Ces évolutions juridiques s'inscrivent notamment dans la loi d'orientation agricole du 9 juillet 1999 et dans la loi d'orientation agricole du 5 janvier 2006. Cependant, le cadre institutionnel agricole reste peu adapté à l'agriculture de service. En particulier, le droit du travail et le régime social et fiscal de l'agriculture se prêtent mal à la prestation de service. Cela peut sembler contradictoire avec le nombre important des politiques qui encouragent l'agriculture de service. Ces politiques se situent à différentes échelles.

À l'échelle internationale, l'encouragement à l'agriculture de service représente l'une des pistes d'adaptation de la politique agricole face à la mondialisation. L'Organisation Mondiale du Commerce (OMC) a défini plusieurs ensembles de types d'aides agricoles selon qu'elles sont soumises ou non à réduction. Les mesures de la boîte verte sont exemptes d'engagement de réduction et se trouvent ainsi encouragées par l'OMC. Elles correspondent à des mesures de soutien 
interne dont les effets de distorsion sont minimes ou nuls sur la production et les échanges. Purement tournées sur le marché intérieur et sans incidence sur le volume de production des denrées agricoles, les politiques en faveur des services environnementaux entrent dans cette catégorie.

À l'échelle européenne, après différentes expérimentations ponctuelles, la politique agricole commune (PAC) a encouragé depuis 1992 le développement de services marchands (visant à la diversification des activités agricoles) et de services non marchands (notamment environnementaux). L'agenda 2000 a mis en place le second pilier de la PAC. Certes, celui-ci ne bénéficie en France que de $10 \%$ de l'ensemble des fonds de soutien à l'agriculture, mais il encourage explicitement la fourniture de services par l'agriculture : notamment la diversification et les services environnementaux (à travers les mesures agri-environnementales).

À l'échelle française, la Loi d'orientation agricole du 22 décembre 2005 a mis l'accent sur certains points relatifs à l'agriculture de service, en particulier le soutien à l'emploi salarié. Mais les évolutions législatives restent modestes en France comparées à celles d'autres pays d'Europe. L'Autriche, par exemple, encourage fortement la fourniture de services par l'agriculture.

À l'échelle territoriale, il existe une grande variété de politiques qui soutiennent l'agriculture de service. Elles sont mises en place aussi bien par les conseils régionaux, les conseils généraux, les parcs naturels régionaux, les structures intercommunales ou encore par les communes. Nous soulignons la grande hétérogénéité de ces politiques. Il existe peu de travaux qui font le point sur les politiques des collectivités territoriales en faveur de l'agriculture de service mis à part les travaux de Berriet-Solliec et al (2004) qui portent sur l'ensemble des aides des collectivités territoriales à l'agriculture.

\section{Conclusion}

Nous avons tenté dans cet article d'apporter un éclairage sur l'agriculture de service, à partir d'un cadre d'analyse issu de l'économie des services. Sur le plan scientifique, il est nécessaire de poursuivre le travail, en particulier pour disposer d'une meilleure description des services produits par l'agriculture. Les dynamiques des marchés de ces services sont encore à étudier car on dispose de peu d'information sur la part respective des agriculteurs et des autres offreurs potentiels de services. Le dispositif statistique agricole peine à rendre compte de ces activités. En particulier, nous ne disposons pas d'éléments permettant d'identifier la nature des exploitations agricoles qui entrent dans une logique de production de service. S'agit-il, conformément à l'idée dominante, principalement de 
petites exploitations à la surface agricole limitée et dégageant peu de revenu ? Comment a évolué dans le temps la production de services par l'agriculture ? Des recherches seraient nécessaires pour répondre à ces questions. Surtout, peu d'études mettent l'accent sur le côté de la demande, pourtant nécessaire pour comprendre l'émergence de marchés de services impliquant l'agriculture.

\section{Bibliographie}

Aznar 0., Guérin M., Perrier Cornet P., 2007, « Agricultures de services, services environnementaux et politiques publiques : éléments d'analyse économique », Revue d'Economie Régionale et Urbaine, $n^{\circ} 4$, p. 573-587.

Berriet-Solliec M., Volay T., Daubard J.-P., 2004, « Les concours publics des collectivités territoriales à l'agriculture en $2000 »$, Notes et Études Économiques, n²0, p. 83-105.

Capt D., Dussol A.-M., 2004, «Exploitations diversifiées : un contenu en emploi plus élevé», Agreste Cahiers, $\mathrm{n}^{\circ}$ 2, mars, p. 11-18.

Clark C., 1940, The conditions of economic progress, rééd. 1957, Macmillan, Londres, 707 p. Gadrey J., 2003, Socio-économie des services, Repères, la Découverte, Paris, 128 p.

Jeanneaux P., Aznar O., Déprés C., 2011, « Les services environnementaux fournis par l'agriculture et leurs modes de gouvernance : un cadre d'analyse économique », Régions \& Cohésion, vol. 1, n³, Winter, p. 117-144.

Kaul I., 2001, "Public goods: taking the concept to the twenty-first century", in Drache D. (dir.), The Market of the Public Domain, Routledge, London and New York, p. 255-273.

Lancaster K. J., 1966, “A new approach to consumer theory”, The Journal of Political Economy, vol. 74, n² 2, April 1966, p. 132-157.

Moati P., 2008, L'économie des bouquets. Les marchés de solution dans le nouveau capitalisme, Ed. de l'Aube, $158 \mathrm{p}$. 